\title{
GEOMATICS AS A SURVEY TOOL TO DOCUMENT AND ENHANCE THE CULTURAL AND LANDSCAPED HERITAGE OF THE MONUMENTAL COMPLEXES IN THE MOUNTAINS OF ABRUZZO
}

\author{
C. Palestini ${ }^{\mathrm{a}}$, A. Basso ${ }^{\mathrm{a}}$ \\ a'Dipartimento di Architettura, Università degli Studi “G. d'Annunzio" Pescara, Italia, \\ caterinapalestini@libero.it - alessandro.basso77@gmail.com \\ Commission VI, WG VI/4
}

KEY WORDS: Eremo, Cultural Heritage, Photomodelling, Laser scanner, image based system, Drone survey

\begin{abstract}
:
The themes of the conference provide an opportunity to exchange views on topics of study in which multidisciplinary contributions of geomatics and restoration contribute to the cognitive process aimed at the conservation of cultural Heritage. In this regard, the contribution exposes research aimed at understanding the documentation and the enhancement of unique architectural - landscape patrimony kept in the Abruzzo mountains. It is about the numerous spiritual retreats established by Pietro da Morrone, Pope Celestino V, mounted among unpassable rocky walls, where the architecture blends with its natural environment camouflaging with it. The analysis refers, specifically to the aspects of survey conducted during the years with the aid of integrated methodologies, able to allow the acquisition, management and comparison of the data. The analysis refers, specifically, to recent digital acquisitions involving the development of San Bartolomeo in Legio, on the slopes of Majella near Roccamorice detected with the use of comparative Agisoft Photoscan and Pix4d software, with shots taken with drones of different sizes, able to mount professional photographic cameras and associate to each picture the coordinates Gps of the point of shooting. Follows a confrontation between a survey carried out with 3d laser scanner, Faro Ls1105, and described acquisitions, obtained from ground and from drone with Photoscan, in order to compare the two scans and the metric differences obtained with the two methods.
\end{abstract}

\section{INTRODUCTION}

The contribution confronts the acquisition of three-dimensional data image based, obtained from photographic images, with those range based images, derived from 3D laser scanner derivates, applying them to a peculiar case study, that of Eremitic structures, in which the architecture blends with the natural environment to which it belongs. Survey thus generates a dual comparison, deriving from the object of detection issues, related to the architecture and the landscape, in the specific case closely connected between them. The large coenobitic presence in Abruzzo linked to the charismatic figure of Pietro da Morrone, Pope Celestino V known for his renunciation to the pontificate, maintained only for a few month in 1294, required in first place the understanding of the hermitic phenomenon and the anthropological motivations that have generated and amplified it within the territory, proposing a first identification and documentation of cultural and physical type. The planned study of different depth levels, from the recognition phase necessary for the knowledge and the documentation of the hermit heritage, of which the recognition is requested in an ongoing project, for the inclusion in the UNESCO heritage site, was then directed towards the aspects of the survey, typical of the geomatics aimed at the preservation.

\section{IMAGE BASED SYSTEM FOR THE ELEVATION OF THE HERMIT STRUCTURES}

The use of systems that operate with the photograph, using passive sensors and exploiting the light present in the surroundings for the acquisition of the images to be processed, not requiring the use of metric rooms, but of common digital cameras, has allowed to take shootings from drone. This latter

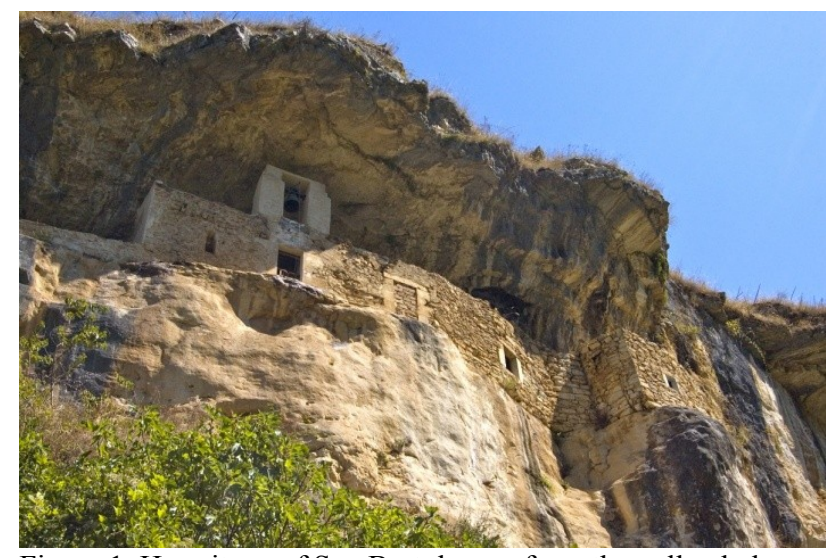

Figure 1. Hermitage of San Bartolomeo from the valley below

resulted particularly useful for reaching the steep walls where they have the hermit structures, hardly detectable with other instruments. The analysis that is proposed refers, specifically, the recent digital acquisitions regarding the complex hermit of San Bartolomeo in Legio, on the slopes of the Majella near Roccamorice, in the province of Pescara, detected with the use of the software Agisoft Photoscan with shots taken from drone with professional cameras, capable of associating to each photo the location GPS of the shot point. The orographic complexities of the places and the technical difficulties associated to the narrow passageways have required an appropriate detection project to determine the choice of the most suitable drones to organize the flight plans of the aircraft systems adopted. The longitudinal prospect anchored to the steep slopes and inaccessible ridges, concealed at the top by a rocky outcrop, was 


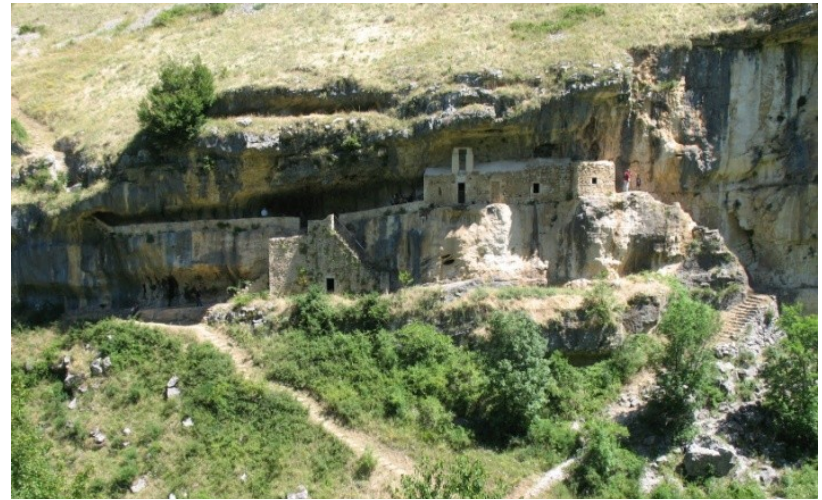

Figure 2. View from the valley in front of the hermitage

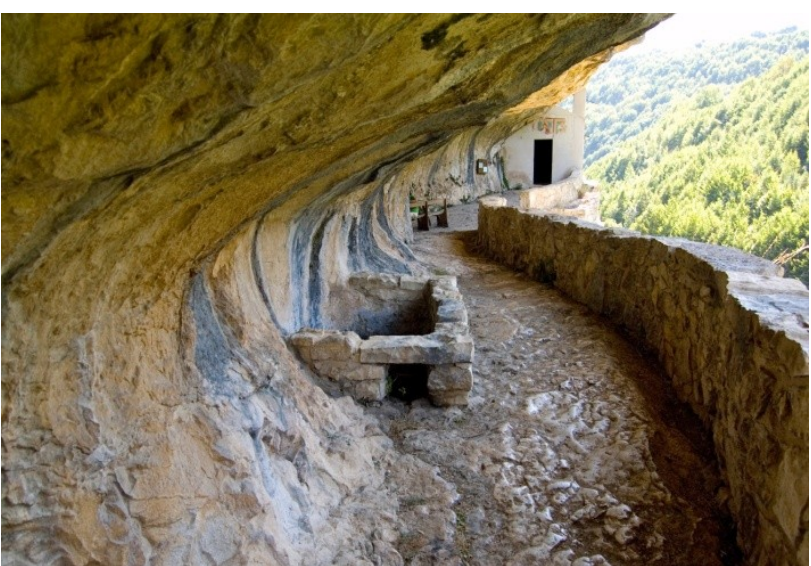

Figure 3. Hermitage entrance from the upper walkway

shot by frontal flight, perpendicular to the facade, not being visible with zenithal views. A comparison was then carried out between a previous survey with 3D laser scanner, Faro LS1105, and the described acquisitions, obtained from ground and from drone with Agisoft Photoscan, in order to compare the two plots and the measurement differences obtained with the software. Photoscan has provided satisfactory results in comparison to the two shooting systems, allowing the creation and the exportation of Dense Point Cloud, triangular mesh and highquality texture, allowing to build a 3D model with texture. The $3 \mathrm{D}$ model of transformation and construction procedure takes place in four main phases based on the calculation principles of the Structure from Motion. The first is formed by the alignment of the camera, in this phase Photoscan analyses all the photos by searching for common points, for each individual imagine and then orientation of the camera and the calibration parameters, from which also it obtains the lens distortions. By this processing there is a cloud of sparse points and the series of shooting positions. The second phase, obtained after orientation, manages the construction of Dense Point Cloud obtained on the basis of the position of the estimated shooting point, from where the program extracts all the possible information. The Dense Point Cloud can also be modified and classified before export or before proceeding to the $3 \mathrm{D}$ mesh generation. The third passage is that of the construction of the mesh, the software converts the cloud of points in a polygon model, reconstructing through 3D polygonal mesh the surface of the object on the basis of Dense Point Cloud. Alternatively, it is also possible to use the Point Cloud method based for the generation of fast geometry, based on the cloud of scattered points. It is useful to point out that, unlike the Laser Scanner that returns a cloud of "orderly" points obtained by a step of scanning format of rows and columns, the image based systems create a very dense network of points, but "disordered". In these cases, the mesh generation algorithm produces uneven results, taking shape on the basis of its own internal logic, produced by the succession of photographic scanning areas that it analyzes. It may be necessary, therefore, to modify or make some corrections to this dense network of points, decreasing the polygonal mesh density, removing detached components, or closing some holes: the program also allows to export the mesh and modify them with other 3D external software, for further more complex operations. When there is the mesh, there follows a fourth and final phase which allows to obtain a textured pattern, that is, a geometric model with photographic texture, carried out through the orthographic projection of the resulting color data of the photos used for the photo-modelling applied on the geometry.

\section{CASE STUDY: EREMO OF SAN BARTOLOMEO IN LEGIO IN ROCCAMORICE (PE)}

Passing to the application stage of the research, as anticipated performed on a detected case study with the two systems, range based and image based, then compared, the survey was conducted on hermit complex of San Bartolomeo in Legio, located approximately 600 meters above sea level. The topography of the place presents abrupt altitude jump through steep paths leading to different entrances to the spiritual retreat, camouflaged between two limestone platforms from the valley. The hermitage fits into a cavity formed under a huge rocky spur that encloses it, concealing its presence, and it allows the entrance via 4 steps, two at both ends and two at the center of the construction, developed along the North West-South East routes coming from the top, located in the South, it is possible to pass through an opening, of around 1,10 meters, at the lower platform leading to the hermit environments. A water collection tank, from the rocky wall, introduces into the walkway (of variable width between $1.00 \mathrm{~m}$ and $1.50 \mathrm{~m}$ ) which are combined the other two stairwells, coming from below (from an altitude of about $-7.5 \mathrm{~m}$ ) arriving at the center of the front terrace to the religious hall, in maximum amplitude point (of about $4.25 \mathrm{~m}$ ). The corridor ends, on an aside, with the front of the small church, now decorated with a labile fresco, and then goes completely in a designed place for worship and two concatenated living spaces that reach to the far end opposite of the terrace, that was once completed by the steep stairway located on the North side. The prospect of the hermitage is visible, in a very foreshortened view, from the underlying part or moving away and reaching the opposite valley which shows a frontal view camouflaged in the landscape. The description of the places helps to understand the difficulties of taking photos and of transport of used equipment, as well as the definition of the adopted survey projects for the two methods.

\subsection{Laser Scanner Survey}

The different orographic levels affect the laser scanner shooting, considering the aforementioned problematic transfer of the instrumentation, from transporting to arrive in the steep paths that lead to the upper walkway and the valley below, with set stations to optimize the views of the hermitage. Contextually the positioning of the removable stations were prepared for placement of the spheres, among them progressively numbered so as to obtain the correct sequence. The acquisition phase performed with the 3D Faro LS1105 laser scanner, for each scan range disclosed its acquisition matrices, the coordinates $\mathrm{X}, \mathrm{Y}, \mathrm{Z}$ for each beat point; the coordinates of each detected point within the acquisition matrices and the relative reflectance value. As usual and then followed the union of scans and their georeferencing in a unique reference system. 


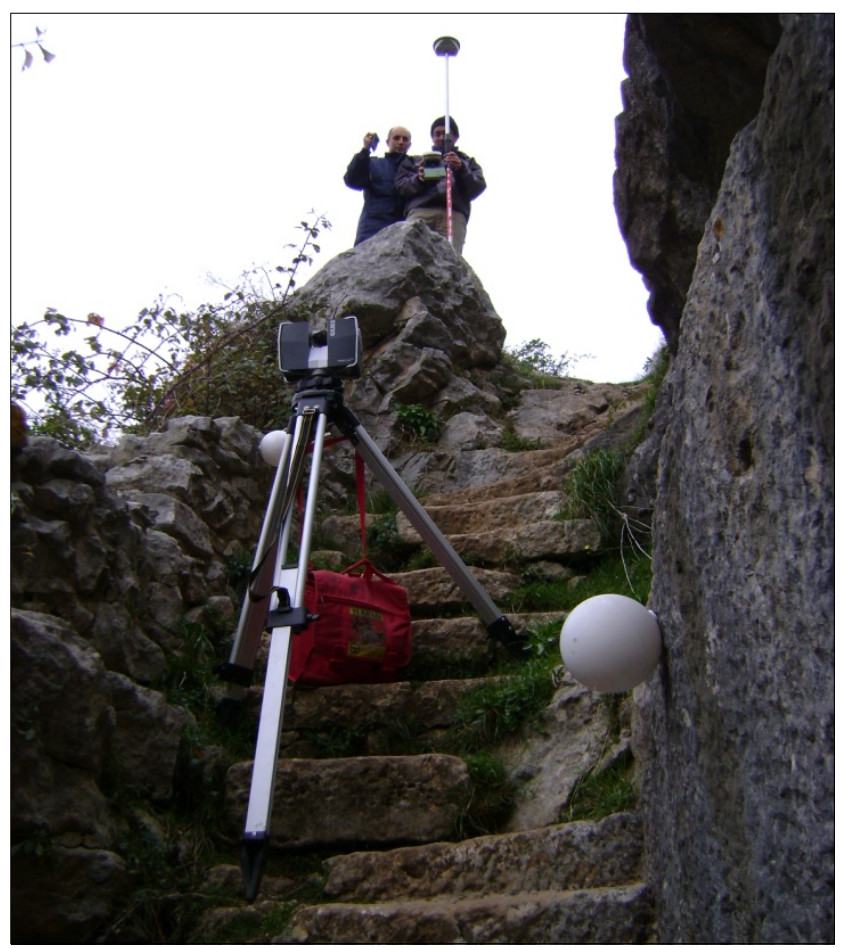

Figure 4. Laser scanner phases

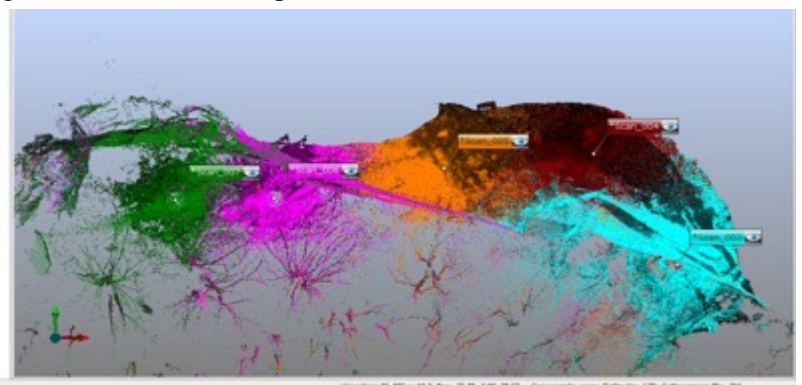

Figure 5. Station points
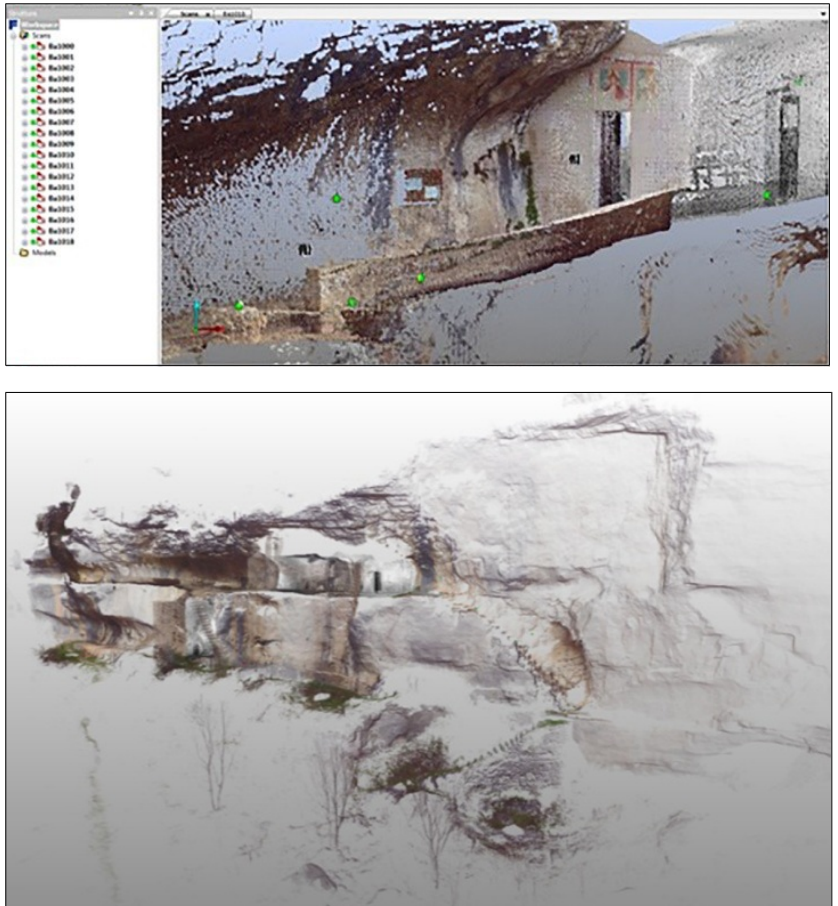

Figure 6-7. Eremo di San Bartolomeo in Legio, regular point cloud
The verification and the acquisition of the measures, initiated the overall process of recomposition of the performed scanning, in plan and elevation view, to then proceed to export the entire point cloud. From these reconfiguration operations of the metric data which, as is known, are already able to offer, in snapshot mode, realistic three-dimensional views of the places, the subsequent graphics processing is delineate, obtained by the arrangement of the section plans, capable of providing the representations of the selected portions using the scan data exportable in various extensions. The graphics show the process of survey described, the filming carried out which gave rise to clouds of points that returned, also through orthophotos, the places and the characteristics, formal and dimensional, of the eremitic structures, permitting through appropriate views and rotations, to examine in detail the melting between the architectural organisms and the rocky walls.

\subsection{Survey image based with the help of drone}

Passing to the image based system, the photo shoots were organized in two distinct sequences, one realized to the level of the terrace and of the walkway that leads to the church, with stations arranged in a concatenation that follows the paths, extending parallel to the wall that delimits up to focus the façade, then proceeding in the interior spaces. The second succession has, instead, considered the view of the longitudinal front, shot with drone of small dimensions, appropriate to shoot places across narrow passages, equipped with a camera type Gopro, able to record videos in full hd, from where frames were extracted. The harshness of the site, with cramped spaces for takeoff, the landing and the capability to perform the shootings safely, made the decision to opt for a manual flight plan, perpendicular to the façade plan, at 10 meters of distance, with passages carried out in the longitudinal direction at different heights in order to cover the entire surface. There was thus obtained the footage from which frames of 1080x1920 were extracted, obtaining a GSD calculation equal to $1.2 \mathrm{~cm} /$ pixel, useful for the elaboration of the point clous. The land pictures integrated the shootings with georeferencing which allowed to scale the points of cloud processed by the software For the flights done by drone, some rules were taken in consideration that allow us to determine the flight height of the drone, how often to take pictures (basic photos taken) and the variable distance of scan progression pass, depending on the scale of restitution. In the hermitage case, the height of flight is equivalent to the distance of the outlet, in other words the distance between the drone and the hermitage, the zenithal views were excluded, since not being visible from above, effecting steps perpendicular to the rocky slope in which the façade inserts.

The method used for the determination of the frame scale, understood as relation between the maximum dimension of the frame (1) and the corresponding real dimension of object (L), called embracement, is based on the formula:

Scale $=1 / \mathrm{L}$

Not being known $\mathrm{L}$, we proceed with an equivalent ratio based on a geometric evaluation, considering the gripping scheme (1a),

from which the formula: $1 / L=p / H$.

The experience demonstrates that generally a relation between the design scale and the frame one is included between 2 and 4 . For example, for a survey that requires a restitution to a $1 / 300$ scale, a medium frame scale is considered about 1/900, a medium scale frame is considered about 1/900 (adopting the mean value as the ratio 3 ). 
The scale is a fact that is established at the start, in function of the requested results and so it has not to be varied like this as the focal length $\mathrm{p}$, the variable value remains at the height of flight $\mathrm{H}$, or in front flight case, the distance of the object to take over D (as illustrated in the scheme 1B), so considering that $P / H=1 / N$

Where $\mathrm{N}$ represents the value of the frame scale to respect. We can find out the fight height:

$\mathrm{H}=\mathrm{P} . \mathrm{N}$

Where:

P: principle distance or focal length

D: shot distance

O: the centre of projection

1: dimensions of the camera sensor

L: Embracing

If considering a focal distance $\mathrm{p}=80 \mathrm{~mm}$, the camera would have to therefore be disposed at a distance $\mathrm{D}=72$ meters. Remembering another principle base where each point must be present in at least two frames shot by two different projection (o) we introduce the concept base of scan distance between two consecutive shots. Until the same points of an object are shot in two consecutive frames, it is necessary that these present an area of overlap where both contain the same object portion, the magnitude of this portion is called longitudinal overlap, and it has an inferior value to 1 and it is indicated with $\mu$.

The longitudinal overlap is strictly satisfied when $\mu=0,5$ $(50 \%)$. The scan distance is therefore that date that permits us to have the longitudinal overlap desired:

$$
B=l \cdot D / p \cdot(1-\mu)
$$

In figure (1c) the distance B is represented, called base shot .In flights by drone, in analogy to air flights, as well as establishing a base, is necessary to also set a wheelbase, between the various strips, in order to guarantee that there is also an overside $\varepsilon$ that usually varies by $10 \%$ to $20 \%$. The calculation of the overlap and overside shot distance permits us to make a survey without the risk of having the areas not properly detected. Another fundamental fact to keep in consideration in an image based system is the GSD (Ground Sample Distance) that indicates to us the survey resolution degree, it expresses the distance between the center of 2 consequent pixels expressed in territorial units, or in other words expresses the "quantity of territory represented by 1 pixel in the "image", it is measured in $\mathrm{m} /$ pixel or $\mathrm{cm} /$ pixel that is determined through a proportion between similar triangles as illustrated in the scheme (1d):

\section{$d: D(G S D)=f: H$}

Where:

$\mathrm{d}$ : pixel dimension

$\mathrm{f}$ : focal distance

$\mathrm{H}:$ shot distance

$\mathrm{D}$ (GSD) : ground sampling distance

So:

$D(G S D)=(d H) / f$

Generally the GSD value for a good job should be less to $5 \mathrm{~cm} /$ pixel.

The drone o Sapr (aeromobile system or remote pilotage) is an aerial platform that can be equipped, depending on the load capacity directly dependent from its dimensions, with a RGB digital camera, with a system of thermal infrared cameras, or with Lidar Systems (www.riegl.com ).

In this case study, focused on survey image based, a drone Chroma Yuneec was used, of small dimensions, with 4 motors, useful to transport of the Gopro. Because of its reduced dimensions, it was the most suitable to detect perched Hermetic Structure, and it also allowed to see the shooting by video.

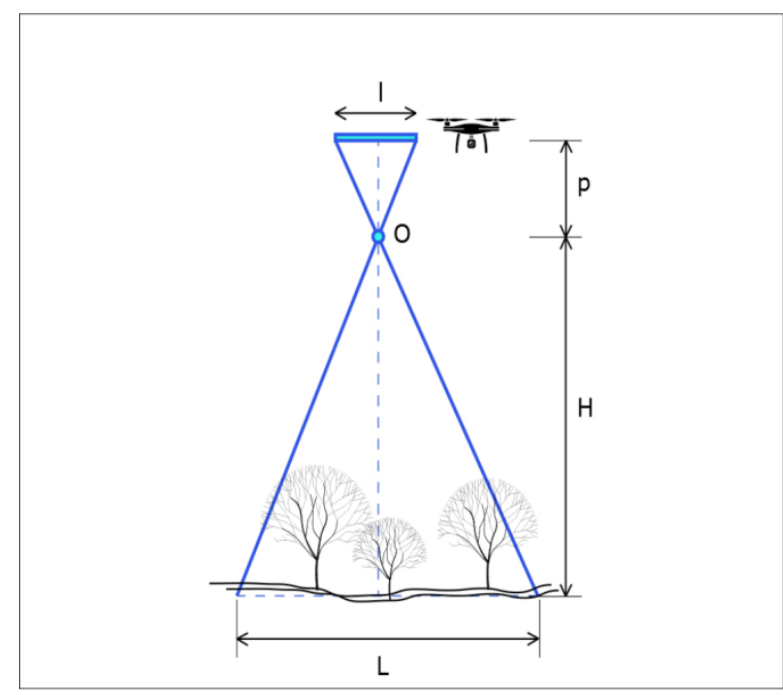

Figure 8. Scan zenith scheme (1a)

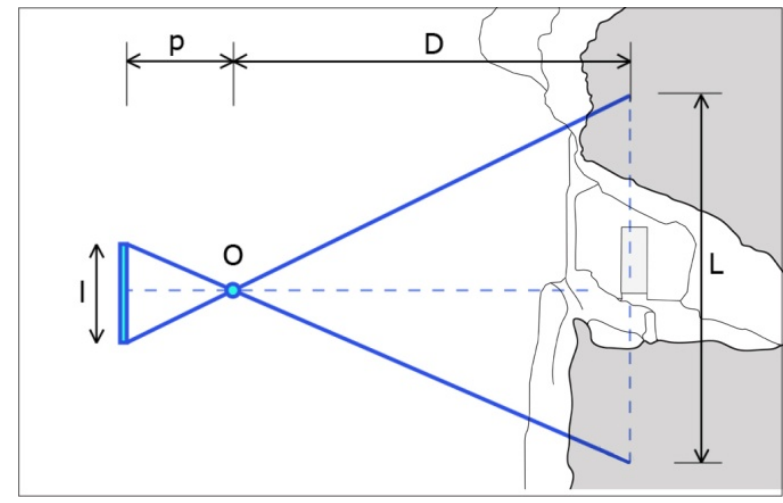

Figure 9. Scan front scheme (1b)

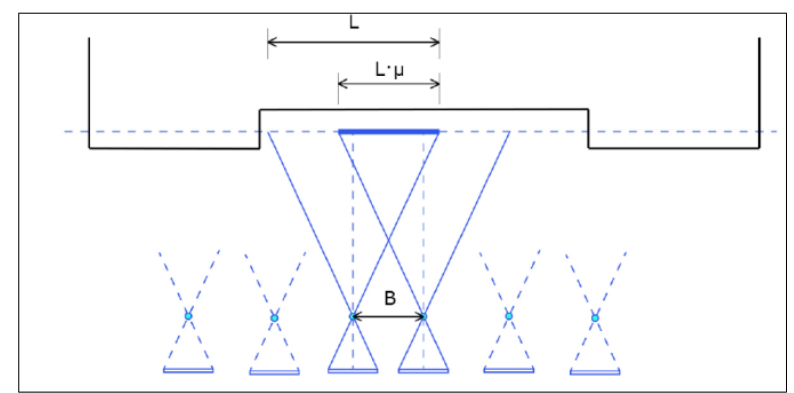

Figure 10. Basic scheme scan, distance between shots (1c)

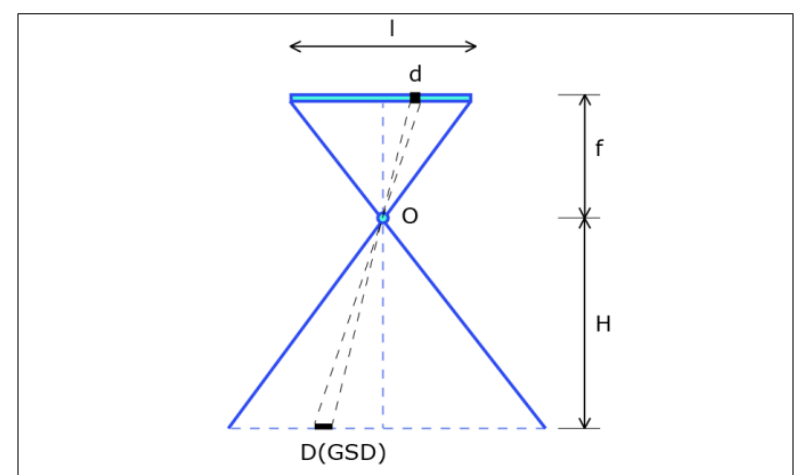

Figure 11. Remote sampling scheme on the ground (1d) 
In take-off phase the weight of this drone is of around 2 kilos with a 20 minute flight autonomy in normal no wind conditions and with a regular flight. As in aerial photogrammetry, where the cameras on board the planes are mounted on suitable stabilizers, also for drones there are balancing systems and anti vibration suspension called "gimbal". Obviously there are chosen on the basis of used camera and will have to be regulated and set by the operator. The stabilizers are able to control and if needed, correct the inclination of the camera in the three axes $\mathrm{x}, \mathrm{y}, \mathrm{z}$ in this way the same angle of inclination will be guaranteed for all the work independently from the oscillation from the drone itself. From the special rubber pieces, they allow to dampen all the oscillations and vibrations of high frequency that are generated during the flight operations. Through a radio, the operator on ground is able to control and manage the flight of Sapr and always through the same, he can manage the stabilizer of the camera in a way to allow, if necessary, to manage during the flight the inclination of the camera itself. Another possible function through the radio is that of taking photos and start or stop the video recording. The principle advantage in the use of drones for survey works is linked to the possibility to scan again parts not visible from ground or difficult to reach, flying on low speed in proximity to objective to take photos. Through the help of cameras made up of focals at variable pixels dimensions, it is possible to obtain images of high resolution, with a Ground Sampling Distance (GSD, dimension of pixel on ground) of order of centimetres. Therefore it appears evident the advantages that may ensue within the image based detecting and photomodelling, wherein in order to survey an object is necessary to take a series of photos captured in a manner to have a prearranged sequence with certain distances and angles.

\subsection{Survey and processing phases}

From the video obtained from the flight, there were obtained about 300 frames, useful for processing points of cloud on the outside of the hermitage, where there are added the photos taken from the ground, from the walkway and interior spaces where a $7 \mathrm{~S}$ alpha Sony photocamera was used, taking about 500 georeferenced photos to 12 megapixel. The photos were then imported into Agisoft Photoscan Pro software (http://www.agisoft.com/ ) (Aicardi et al., 2016) to start the alignment phases and generating points of cloud. The complex shape of the place required the use of three "chunks", one for the outside and two for the interior space, in particular for the chapel and for the planted rearward living environments, then merged into a single file PhotoScan. The adopted subdivision offers the possibility to accurately manage the cloud of points relating to the different compartments of the construction, in particular sessions impossible to manage because of the inconsequential morphology and variable light exposure conditions of the photos used. To allow the optimal fusion of the mesh, the operation of detection of visible marker was performed, positioned both inside and outside, through where was possible to reconnect the two internal sessions between them and then the external one, also obtaining the measures relating to the thickness of the rock and the perimeter walls. The operation of union of chunks previous to the calculation of the mesh, results to be more precise because the software, working on photogrammetric data directly contained in the points cloud, determines with accuracy each marker individualized by homologous points of a pair of photos, an operation that in the next step, in which the program has already elaborated a continuous three-dimensional surface, may generate polygonal interpolation errors and pattern matching, due to a different topology automatically and separately generated during the phase of meshing of points cloud.

From the cloud of points we proceed to the mesh calculation, phase in which it is possible to intervene on the density of the polygonal mesh, in basis of the desired visual quality but also in relation to the scale of the survey requested. In this phase it is very frequently possible to incur in artefacts or in errors of definition surely caused by the complexity of the scenario but very often by the digital noise, like the vegetation, that in photographic shoots tend to cover part of the structure, causing then gaps and imperfections that make the next phase of texturing. A series of algorithms of correction are already present in the Agisoft product but it is preferred to proceed exporting the model on the external softwares that are specific as MeshLab (http://www.meshlab.net ) and Autodesk Remake (https://remake.autodesk.com/about ) active to a fast and dynamic control on the polygonal surfaces that present critical issues. The last phase is about the application, through a projection system, of the texture on three-dimensional models created and provided by UV Map, taking advantage by photos already used for the photo - modelling. A diffuse map and a Normal map are therefore generated, necessary to a detailed and photorealistic return of the three-dimensional model obtained, exportable and implementable on external platforms of precalculated rendering of RTR (Real Time Rendering). The images accompanying the text document the phases described, also present a series of vertical sections and also horizonatal ones obtained from survey, replicable in the desired points, that as in a TAC system can identify a peculiarity of the simple architecture of the hermit closely connected with the rocky walls. These last ones obviously present many irregularities linked to the ancestral constructive techniques, being in many points dug by the rock, of different thickness, and also the acquisitions refer contextually to the landscape in which the eremo fits itself camouflaging itself with it. The work of the drone permitted to detect points very difficult to reach and acquirable with other methodologies, even $3 \mathrm{~d}$ laser scanner where the confrontation is proposed.

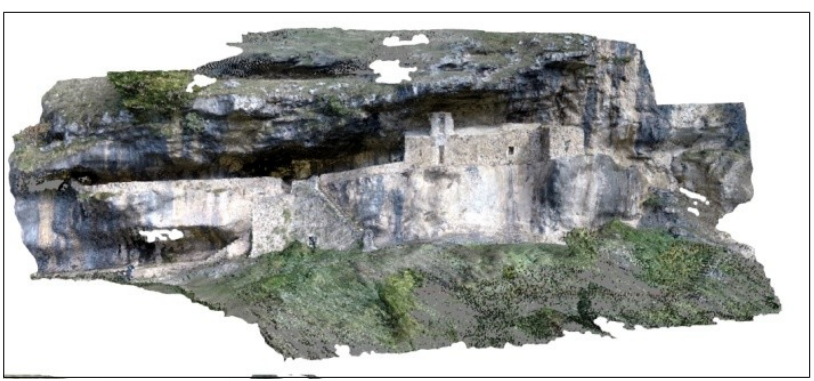

Figure 12. Image Based Point Cloud

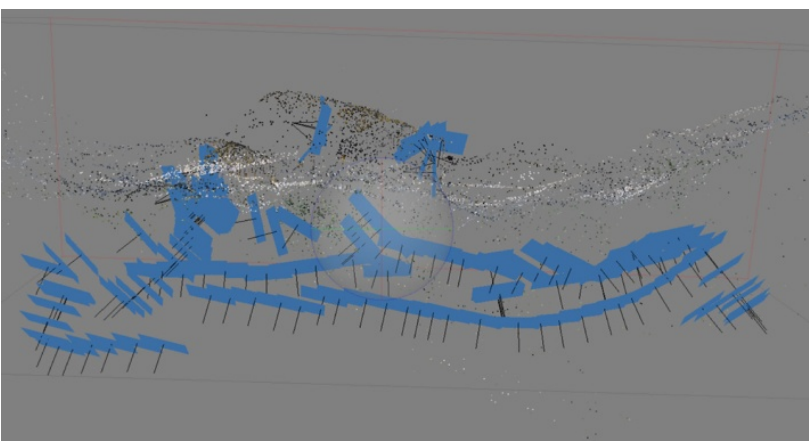

Figure 13. Shooting scheme 


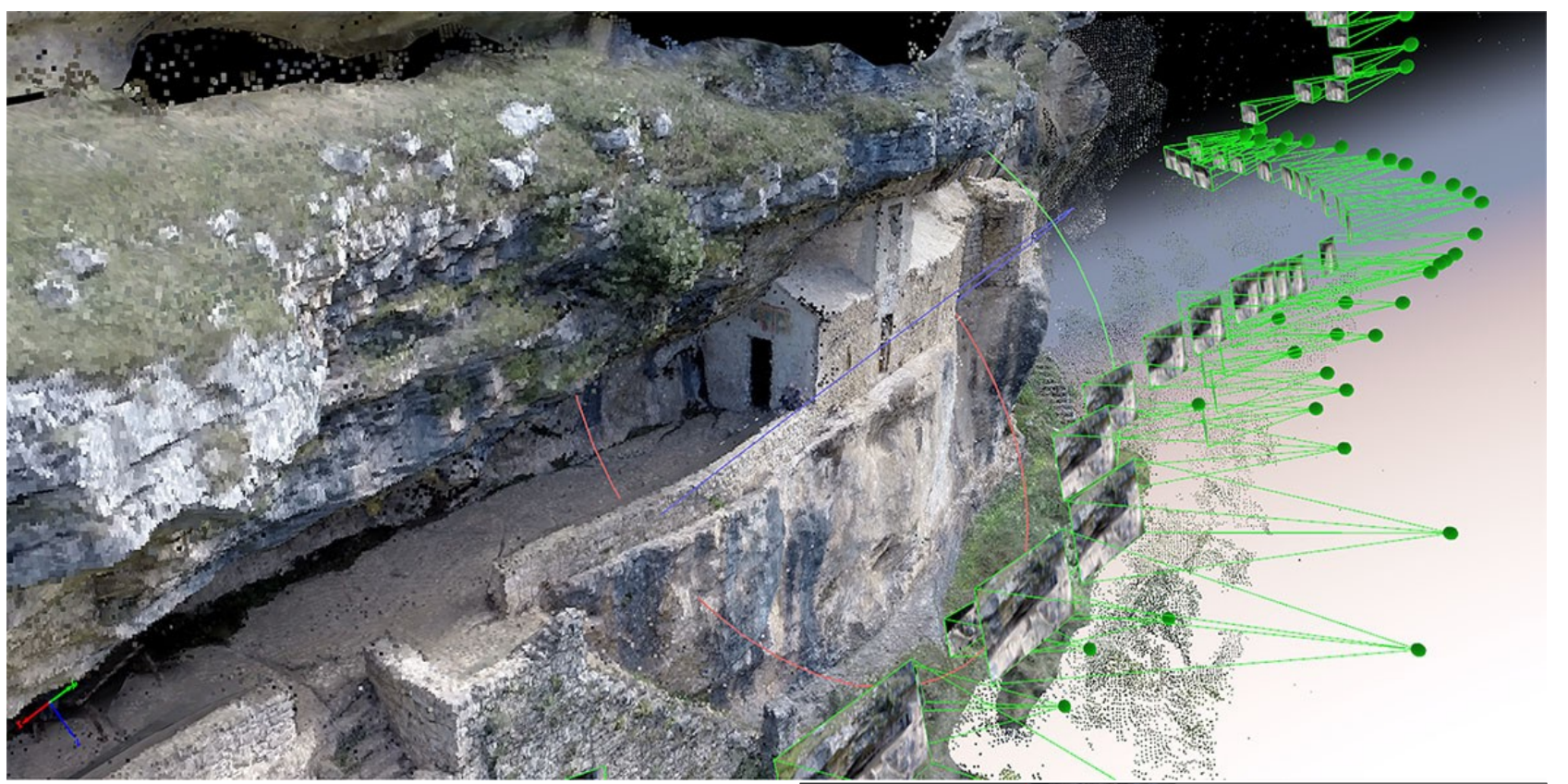

Figure 14. Drone shooting scheme ( Points Cloud)
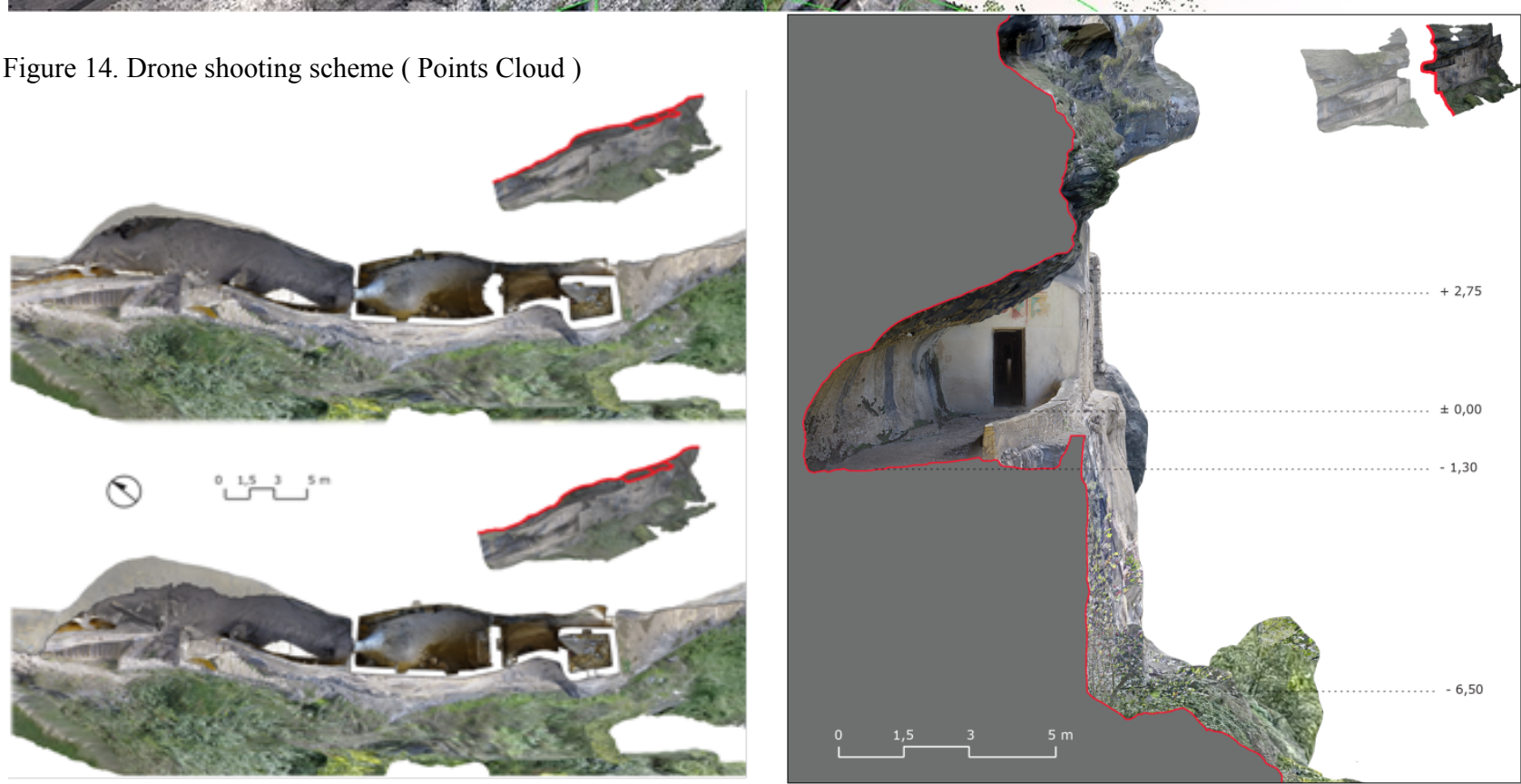

Figure 15. Horizontal sections

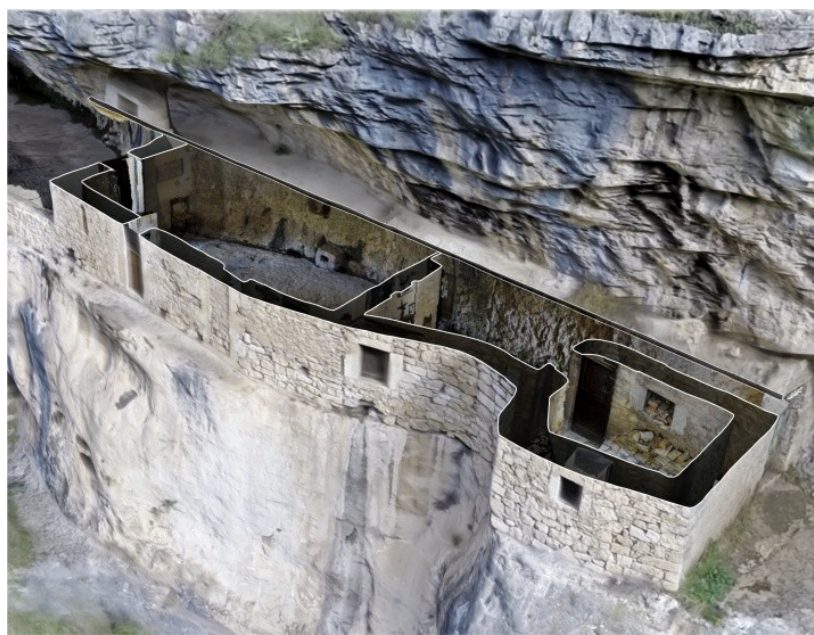

Figure 16. Vertical sections
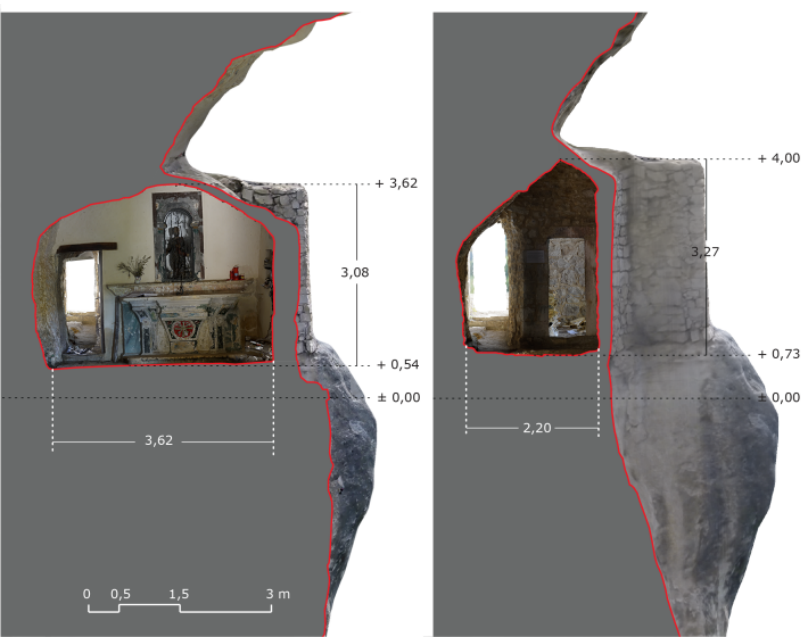


\subsection{Comparison of detection systems Range Based - Imaged Based}

There was finally done a comparison between the two systems of survey to test the validity and the obtained results in the specific case study. For this purpose and with the aid of software Cloud Compare (http://www.danielgm.net/ccl ) (Girardeau - Montaut, 2013), two point clouds were confronted, that elaborated by software Photoscan through the flight with the drone, and that obtained by the laser scanner acquisition. The first step was that of importing the different clouds in the software to perform the alignment of the both, done through the overlap obtained through the convergence of three well visible points on both the clouds. When the three points were identified, the software proceeds in an automatic way to perform the alignment. If the points of imported clouds present a different scale, the same program will perform a scale operation, bringing the clouds back to the same scale without any deformation. In a first visible analysis of the clouds, it is already evident that the elaborated cloud of acquisition laser scanner results to have several gaps for zones lacking data, relating to non revealed parts, among these: coverage, obviously not accessible by the share ground, but also many parts of longitudinal elevation. This is attributable to morphology of the site that doesn't allow to use all the necessary stations to shoot the front in a correct way, except with very foreshortened view from below or too camouflaged with the landscape in distant shooting from the opposite valley. To a more detailed analysis with numeric feedback provided by the software, the points of cloud of laser scanner results to be much more sharp in respect to the acquired cloud with Photoscan and elaborated with the technique of photomodelling. The cloud of the laser scanner results to have around 150 million points, while the one elaborated by Photoscan has around 5 million points.

Despite this numerical difference, the acquired cloud by drone results to be much more complete with less gaps. Next it proceeds to the alignment of the different clouds to have a reply in numerical terms and to quantify concretely measures errors and differences. About that, 3 points were taken as is seen by the image (A) marked with: R0, R1, R2, the obtained result gives an error in R1 between the two clouds with a rest of 3,12 $\mathrm{cm}$. For the complexity and the extension of survey it can be considered acceptable an error of these dimensions, considering also that the cloud elaborated by Photoscan by drone acquisition seems to be much more complete in respect to that elaborated by the laser scanner that is sharper and more precise, but lacking many parts. The images show what is exposed, documenting the relative passageways.

\section{CONCLUSIONS}

In conclusion the laser detection offers more detail in areas detected from the ground, specifically in lower part of the valley and in some portions of passageway. The comparison carried out permitted to observe that the image based system results to be an important work instrument, considering the low costs to use it and the possibility to obtain tri-dimensional scansions by common digital photocameras. In summary, over the methodological considerations on the survey purpose, that lead to the choice and conduct appropriate integration of suitable systems to achieve its objectives, with the knowledge that each technique has specific prerogatives, such systems with the appropriate application measures offer a valid alternative within the three-dimensional survey, waiting for the market makes more accessible the LiDAR systems laser scanner, assembled on drones, light and suitable to the movement.

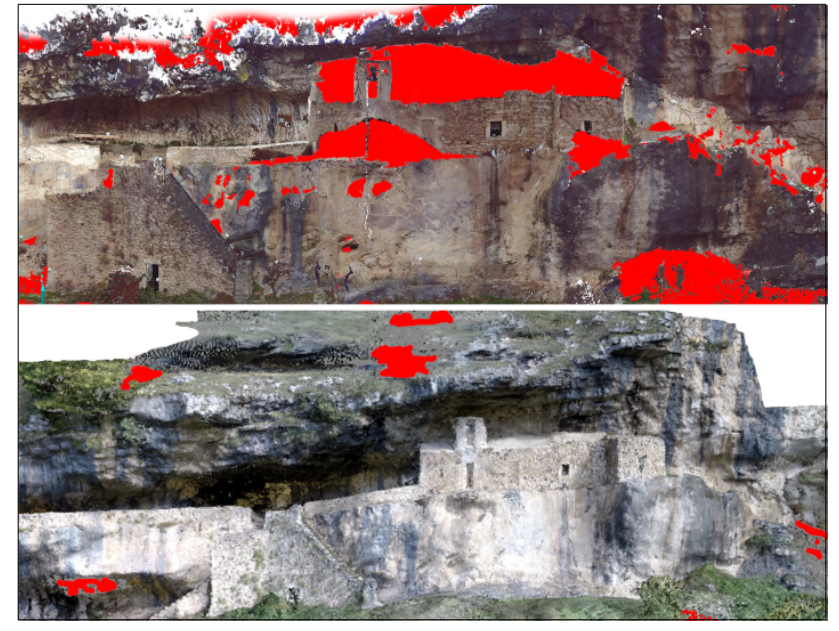

Figure 17. Compare point clouds, in red missing parts
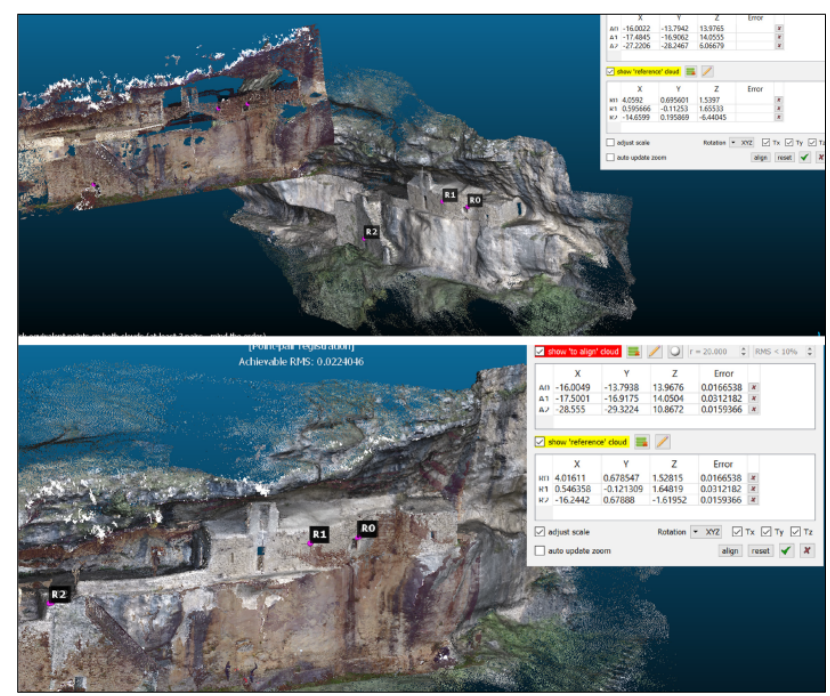

Figure 18. (A) Alignment phases with numerical control

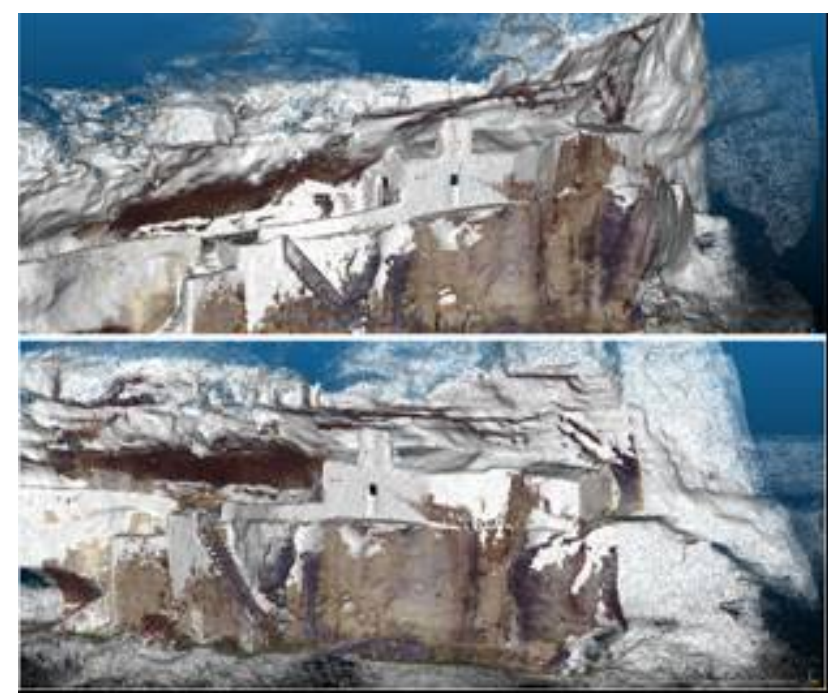

Figure 19. Overlapping point clouds: in white shader, based image acquisition 


\section{ACKNOWLEDGEMENTS}

The authors wish to thank in particular the people involved in the acquisition and management of data and in the project on site among which the architects Alessio Grugnale for the acquisitions laser scanner, Fabio D'Ercole for the elaboration of image based data and Matteo Spinozzi for the shootings on drone.

\section{REFERENCES}

Amadesi, E., 1975, Fotointerpretazione e Aerofotogrammetria. Bologna, Italia: Pitagora Ed.

Cundari, C., 1984. Fotogrammetria Architettonica. Bologna, Italia: Kappa Ed.

De Luca, L., 2011. La Fotomodellazione Architettonica. Palermo, Italia: Flaccovio D. Editore.

Remondino, F., Barazzetti, L., Nex, F., Scaioni, M., Sarazzi, D. 2011. UAV photogrammetry for mapping and 3D modelingcurrent status and future perspectives. ISPRS Archives, 38(1), C22. DOI: 10.5194/isprsarchives-XXXVIII-1-C22-25-2011.

Palestini, C., 2011. Contesti ambientali e strutture architettoniche di insediamenti eremitici in Abruzzo, in Architettura Eremitica Sistemi progettuali e Paesaggi culturali. Atti II Convegno Internazionale di Studi, Vallombrosa 24-25 settembre 2011. Firenze, Italia: Edifir Ed., pp. 89-96.

Palestini, C., 2012. Insediamenti eremitici in Abruzzo: work in progress, in Architettura Eremitica Sistemi progettuali e Paesaggi culturali. Atti III Convegno Internazionale di Studi, Camaldoli 21-23 settembre 2012. Firenze, Italia: Edifir Ed., pp. 266-271.

Palestini, C., 2013. The cultural heritage of Abruzzo's Thebaid, in Heritage and Unesco Sites memory, measure and harmony, $\mathrm{X}$ Congresso UID , Matera 24-26 ottobre 2013. Roma, Italia: Gangemi Ed., pp. 755-762.

$\mathrm{Wu}, \mathrm{C}$, 2013. Towards Linear-Time Incremental Structure from Motion, 3DV 2013 proceedings, pp.127-134.

FARO, 2013. Scene Version 5.1 User Manual. FARO Technologies Inc, Lake Mary FL, section 5.6

Ballabeni, A., Apollonio, F. I., Gaiani, M., Remondino, F., 2015. Advances in image pre-processing to improve automated 3D reconstruction. ISPRS Archives, XL-5/W4, pp. 315-323.

Gaiani, M., Apollonio, F.I., Clini, P., 2015. Innovative approach to the digital documentation and rendering of the total appearance of fine drawings and its validation on Leonardo's Vitruvian Man, Journal of Cultural Heritage. 16(6), pp. 805812.

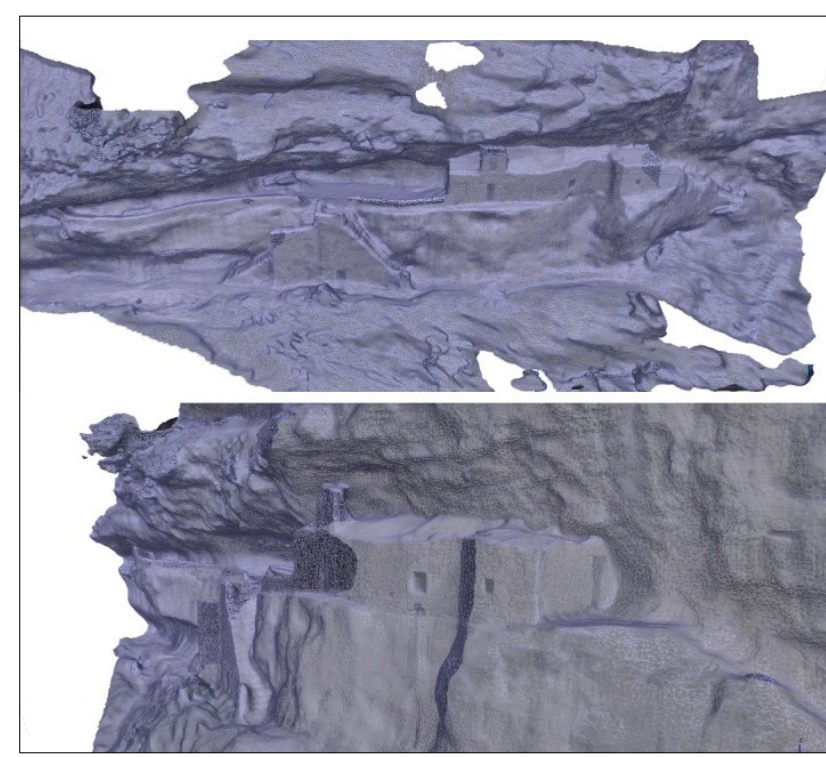

Figure 20. Correction of polygon meshes through specific tools

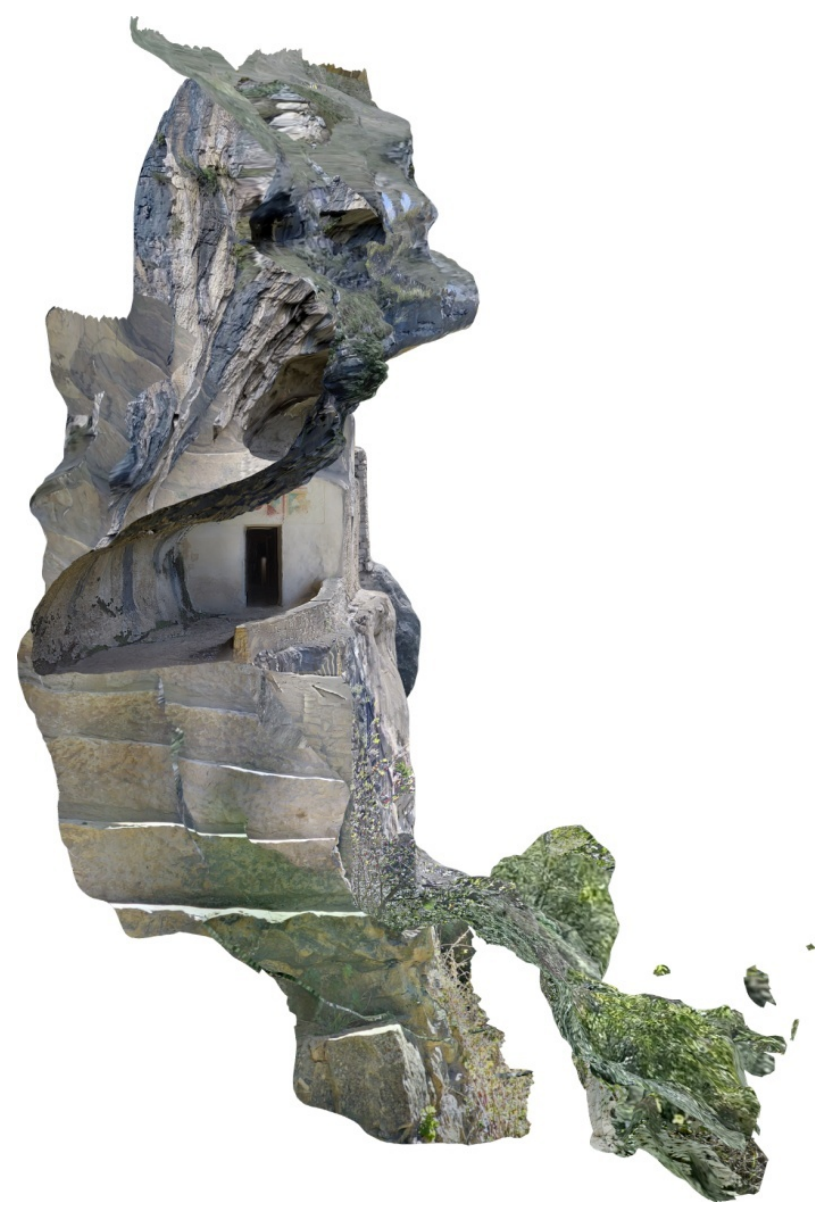

Figure 21. Complete polygonal 3d model, textured and with new geometric topology 